Ruth S. Estimar, MD

Mario Adrian M. Zafra, MD

Ramon Antonio B. Lopa, MD

Department of Otorhinolaryngology

Philippine General Hospital

University of the Philippines Manila
Correspondence: Ruth S. Estimar, M.D.

Department of Otorhinolaryngology

Ward 10, Philippine General Hospital

Taft Avenue, Ermita, Manila 1000

Philippines

Phone: (632) 5264360

Fax: (632) 5255444

E-mail: orlpgh@yahoo.com

Reprints will not be available from the author.

No funding support was received for this study. The authors signed disclosures that they have no proprietary or financial interest with an organization that may have a direct interest in the subject matter of this manuscript, or in any product used or cited in this study.

Presented at the Interesting Case Contest, Philippine Society of Otolaryngology-Head and Neck Surgery Midyear Convention, Baguio Country Club, Baguio City, Philippines April 20, 2007.

\section{Congenital Nasal Chondromesenchymal Hamartoma}

Objectives: To report the case of a congenital nasal chondromesenchymal hamartoma in a oneyear-old female and review the literature, identifying problems encountered in confirming the diagnosis and in treatment of this patient.

\section{Methods:}

\author{
Design: Case Report \\ Setting: Tertiary Public General Hospital \\ Patient: One
}

Results: A one-year-old female with an intranasal mass noted at birth and with subsequent unilateral maxillary enlargement is described. Computed tomography showed calcifications and erosion of adjacent bony structures. Histopathology and immunohistochemistry of an intranasal biopsy were interpreted as chordoma, a malignant tumor. Following surgical excision, the final histopathologic diagnosis was chondroid hamartoma.

Conclusion: Only 20 cases of nasal chondromesenchymal hamartoma have been reported in the literature worldwide. These tumors may present clinically, histopathologicaly and radiologically as malignant tumors and may mislead even the experts. The whole clinical picture should be taken together to avoid misdiagnosis as a malignancy and to facilitate appropriate management.

Keywords: nasal chondromesenchymal hamartoma, nasal masses in infancy, nasal chondroid lesions

Nasal masses in infancy are infrequently encountered. Most are developmental anomalies such as encephalocoeles, gliomas and nasolacrimal duct cysts. The rest are neoplasms, primarily composed of teratomas and dermoid cysts. Occasionally, a variety of benign and malignant soft tissue tumors occur in children. ${ }^{1}$

The majority of head and neck neoplasms in children are benign. Primary malignant neoplasms of the head and neck are not common and account for about $5 \%$ of neoplasms 
occurring in childhood. In children, the neoplasms are more frequently reticuloendothelial, neural or mesenchymal, as opposed to the predilection for epithelial neoplasms in adults. ${ }^{1}$ Chondrogenic tumors of the maxillofacial region may be difficult to differentiate histologically and radiologically as to whether they are benign or malignant.

We present the case of an infant with a nasal chondromesenchymal hamartoma which was confused with a malignancy. This clinicopathologic entity is remarkable not only because of its rarity but also because it may present a diagnostic dilemma with major implications in management.

\section{CASE REPORT}

A term female was born at home with the assistance of a traditional birth attendant to a then 23-year-old G2P1 (1001) mother. Because of cyanosis and dyspnea at birth, the baby was rushed to a local hospital where physicians were unable to pass a suction catheter through the right nasal cavity. Glatzel's mirror test showed no misting from the nostrils, and a whitish mass was noted in the right nasal cavity. Subsequently, chronic mucoid discharge from the right nostril and a progressively bulging right maxilla were noted.

A computed tomography (CT) scan of the paranasal sinuses at three months of age revealed a mixed-enhancing, soft tissue mass at the right nasal antrum with irregular foci of calcifications, measuring $2.2 \times 3.22 \times$ $3.03 \mathrm{~cm}$. The mass pushed the nasal septum medially and the medial wall of the right maxillary sinus laterally. No bony erosions, intraorbital or intracranial extensions were seen. Considerations were an ossifying fibroma, calcifying polyp or dentigerous cyst. Surgery was advised but deferred by the mother.

At eight months of age, repeat CT scans revealed a $3.0 \times 3.95 \times 3.33$ $\mathrm{cm}$ mass, now occupying the right maxillary and ethmoid sinuses with extension in the anteromedial portion of the right orbit (Figure 1). There had been an increase in size of $83.7 \%$ over the past five months.

They finally agreed to admission, and repeat $\mathrm{CT}$ scans revealed a $3.20 \times 4.03 \times 3.4 \mathrm{~cm}$ ethmoidal mass (a $103 \%$ volume increase in 9 months), with lysis of the right lamina papyracea, extension into the right intraorbital extraconal compartment with involvement of the medial rectus and inferior oblique muscle, and thinning of the medial wall of the right maxillary sinus (Figure 2). No intracranial extensions were noted. The radiological impression was an ethmoidal mass with malignant features.

An intranasal punch biopsy was interpreted as a mesenchymal neoplasm compatible with chordoma, a diagnosis later supported by slide review. Immunohistochemical stains were strongly positive to $\mathrm{S} 100$ and vimentin with non-immunoreactivity to cytokeratin. These findings were still interpreted as consistent with a chordoma.

Considering a highly malignant tumor, a right medial maxillectomy

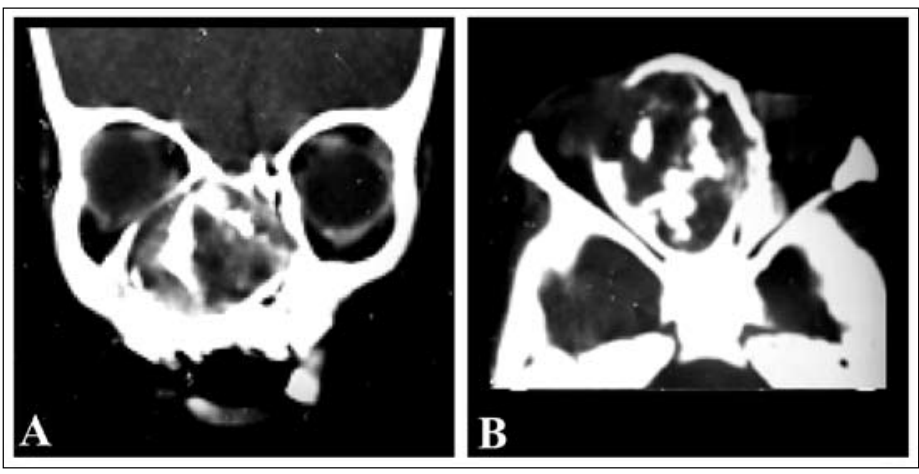

Figure 1. Paranasal sinus $C T$ scan ( $A$, coronal and B,axial cut) at 8 months of age showing an ethmoida mass with extension into the right maxillary and ethmoid sinuses and the anteromedial portion of the right orbit

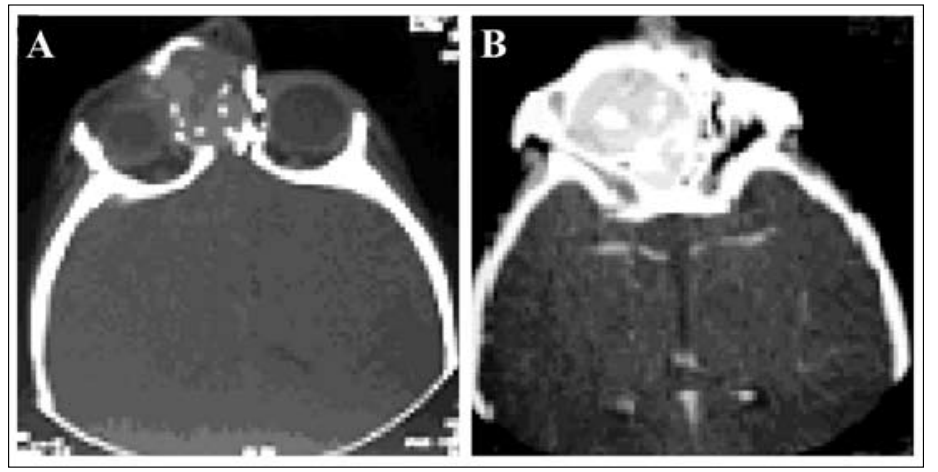

Figure 2. PNS CT scan(A, axial cut, bone window; $B$, axial cut with contrast) at one year of age showing an ethmoidal mass with lysis of the right lamina papyracea and extension into the right intraorbital extraconal compartment and thinning out of the medial wall of the right maxillary sinus

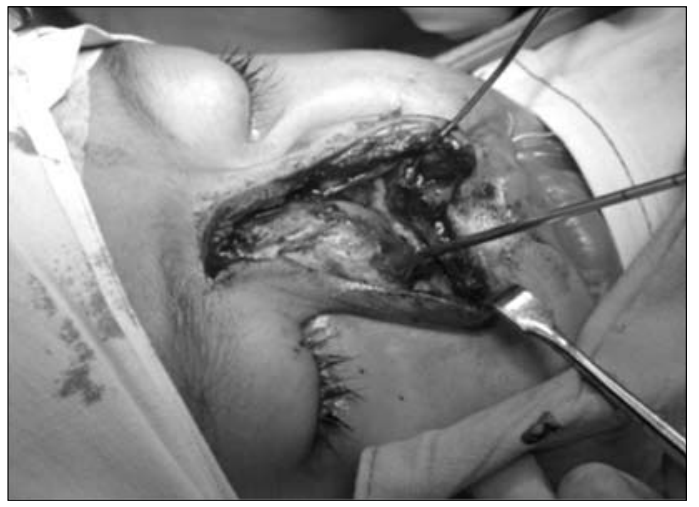

Figure 3. A right lateral rhinotomy with a Lynch extension revealed a well-circumscribed mass in the ethmoid sinus extending to the maxillary sinus and medial wall of the orbit

via right lateral rhinotomy combined with a Lynch incision was performed (Figure 3). Intra-operatively, an eggshell-thin anterior maxillary wall, a whitish mass filling the right ethmoid and maxillary sinuses, and septum deviated to the contralateral side were noted. The orbital wall was intact but the nasal roof showed small thinned-out areas. The patient tolerated the procedure without any problems or post-operative complications.

Grossly, the mass was cream to tan-colored, firm and well- 

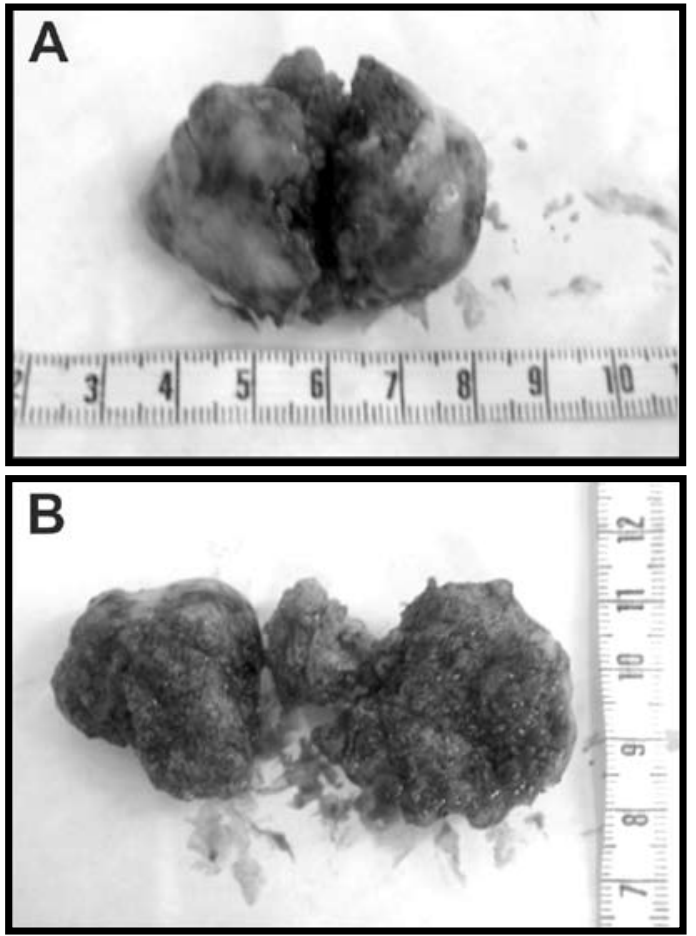

Figure 4. Gross appearance of the ethmoidal mass measuring $4 \times 3.5 \times 3 \mathrm{~cm}$. (a) whole specimen; (b) cut surface

circumscribed, measuring $4 \times 3.5 \times 3 \mathrm{~cm}$. The surface was smooth, but gritty and irregular when cut (Figure 4). No tumor hemorrhages were noted. Histologically, nodules of hyaline cartilage ranging from fairly immature looking chondroid stroma to islands of mature hyaline cartilage were found in a variably cellular stroma that was fibrocytic in some areas and loose and myxoid in others (Figure 5). Bundles of dense collagen as well as occasional spicules of bone were also noted.

The histopathologic diagnosis at this institution was a mesenchymal neoplasm with chondroid differentiation. Further analysis at the Memorial Sloan-Kettering Cancer Center and IMPATH Laboratories New York yielded a final pathologic diagnosis of chondroid hamartoma with osseous metaplasia.

\section{DISCUSSION}

Nasal chondromesenchymal hamartoma (NCMH) is a rare, destructive yet benign, tumefactive lesion involving the nasal cavity and paranasal sinuses. The term was first coined by McDermott et. al. in 1998 to describe a distinct clinicopathological entity composed of a proliferation of mesenchymal and cartilaginous elements. ${ }^{4}$ Nasal chondroid lesions, nasal hamartoma, chondroid hamartoma and mesenchymoma are other terms used to refer to this disease entity. It usually affects infants less than 3 months of age although there have been reports of occurrence in adolescents and young adults. ${ }^{5}$ One case series reports occurrence in a 69 -year-old female. ${ }^{6}$ Presently, there have
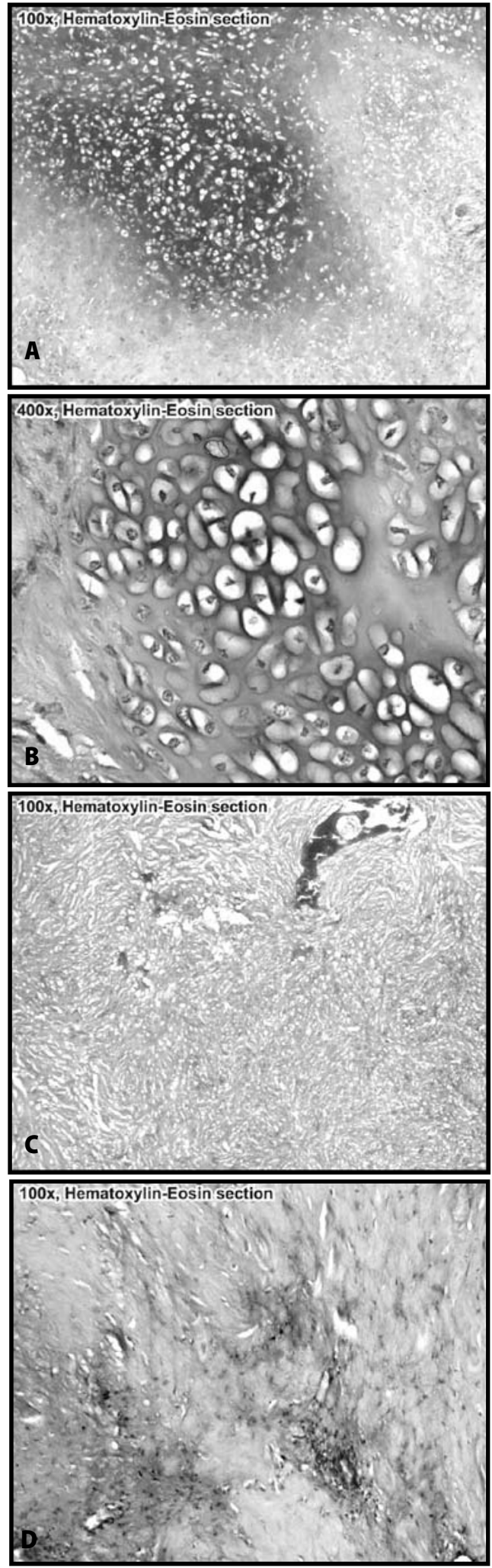

Figure 5. NCMH in this patient showing nodules of hyaline cartilage ranging from (A) fairly immature looking chondroid stroma to (B) islands of mature hyaline cartilage. Stroma is (C) fibrocytic in some areas and (D) loose and myxoid in others 


\section{CASE REPORTS}

only been 20 cases of NCMH reported in English medical literature., ${ }^{4,}$ $7-14$

Due to the paucity of cases, the pathogenesis of NCMH is not well understood. Hamartomas are characterized by an abnormal mixture of tissues indigenous to that area of the body, but with an excess of one or more of the tissue types. The development of hamartomas may involve errors during fetal growth or disturbances of immature tissues in the post-natal period. ${ }^{4}$ It is believed that fibroblasts and myofibroblasts are the major component cells in $\mathrm{NCMH} .{ }^{9} \mathrm{NCMH}$ was initially thought to be present at birth, indicating a developmental or congenital origin. However, with reports of occurrence in adolescents and adults, alternative explanations such as the role of inflammation, cytokines, and growth factors or the association with hormonal stimulation are being investigated. ${ }^{11,13}$

Differentiation between $\mathrm{NCMH}$ and a malignant lesion like a chordoma may be difficult. NCMH have a seemingly infiltrative nature. As such, it may mimic malignant tumors in its clinical, radiologic or even histopathologic presentation. In the literature, presenting symptoms included a nasal mass, nasal obstruction, respiratory distress and a maxillary bulge which were all present in this patient. Orbital involvement can result in proptosis, enophthalmos or impairment of eye movement. Intracranial extension of the tumor can result in neurologic manifestations such as hydrocephalus and oculomotor disturbance. ${ }^{10}$ Our patient had no ocular or neurologic symptoms or actual orbital invasion despite a preoperative CT scan suggesting intraorbital involvement.

Radiologically, NCMHs may be nonencapsulated and ill defined, containing both solid and cystic portions. Calcifications may be present. ${ }^{4}$ The adjacent paranasal sinuses are frequently involved and erosion of the surrounding bone and extension to the skull base and orbital region are not uncommon. In a review of 19 cases by Johnson et. al., ${ }^{14} 67 \%$ demonstrated bony remodeling, thinning or erosion and $53 \%$ demonstrated intracranial extension through the cribriform plate to the anterior cranial fossa. 50\% revealed internal calcifications while $40 \%$ revealed cystic components. In this patient, the rapid growth of the mass, calcifications and destruction of adjacent bony structures were interpreted as consistent with a malignancy.

Biopsy results may also be misleading. Histologically, NCMH is comprised of a variety of mesenchymal components with a focally lobular architecture of irregular islands of hyaline cartilage with occasional binucleated chondrocytes and cartilaginous islands. ${ }^{4}$ Thus, NCMH may morphologically overlap with chondroid lesions including malignancies such as chondrosarcoma and chordoma (Figure 6).

Immunohistochemistry may be helpful if the initial histological picture is unclear. Chordomas, which are tumors arising from embryonic notochordal remnants, and NCMH both stain positive for S100 and vimentin which are markers for neuroepithelial cells and mesenchymal cells respectively. However, chordoma stains positive for cytokeratin while NCMH is negative. ${ }^{9}$ Negativity to cytokeratin in this case should have been a strong reason to discount a chordoma, a diagnosis which was strongly maintained by the pathologist.

Taking all these into account may elucidate how a nasochondromesenchymal hamartoma, a rare but benign lesion, was mistaken for a chordoma, an aggressive and malignant tumor. Accurate differentiation between NCMH and malignant tumors is very important, as a hamartoma's capacity for growth is usually limited and its biologic behavior is typically benign, ${ }^{8}$ making complete surgical excision sufficient therapy. When as in our case, a misdiagnosis of malignancy is made, excessively radical surgery and possibly, potentially harmful neo-adjuvant radiotherapy may result.

Despite its relatively low prevalence, $\mathrm{NCMH}$ should be considered as a differential diagnosis in patients with chondroid lesions in the maxillofacial region, particularly in infants. Caution should be exercised in diagnosing radiologically and histologically aggressive-looking maxillofacial tumors as malignant especially in infants. Ancillary studies may be necessary to further confirm a malignancy. As in all cases, individualized treatment of patients with head and neck tumors is very important. Factors such as age, co-morbid illnesses and location of the tumor should be considered in choosing treatment approaches.

\section{REFERENCES}

1. Thawley SE, Panje WR, Batsakis JG. Comprehensive management of head and neck tumors. 2nd edition. WB Saunders Company; 1999. p. 1843-44, 1876-78.

2. Cummings CW, Flint PW, Haughey BH, Harker LA, Richardson MA, Schuller DE et al. Otolaryngology Head and Neck Surgery. 3rd ed. Mosby-Year Book, Inc; 1998. p. 1212, 3988, 4183 85.

3. Terris MH, Billman GF, Pransky SM. Nasal hamartoma: case report and review of the literature. Int $J$ Pediatr Otorhinolaryngol 1993; 28:83-88.

4. McDermott MB, Ponder TB, Dehner LP. Nasal chondromesenchymal hamartoma: an upper respiratory tract analogue of the chest wall mesenchymal hamartoma. Am J Surg Pathol 1998; 22:425-433.

5. Alrawi M, McDermott $M$, Orr D, Russel J. Nasal chondromesenchymal hamartoma presenting in an adolescent. Int J Pediatr Otorhinolaryngol 2003; 67: 669-672.

6. Ozolek JA, Carrau R, Barnes EL, Hunt JL. Nasal chondromesenchymal hamartoma in olde children and adults. Arch Pathol Lab Med 2005; 129:1444-1450.

7. Kato K, ljiri R, Tanaka Y, Hara M, Sekido K. Nasal chondromesenchymal hamartoma of infancy: the first Japanese case report. Pathol Int 1999; 49:731-736.

8. Kim DW, Low W, Billman G, Wickersham J, Kearns D. Chondroid hamartoma presenting as a neonatal nasal mass. Int J Pediatr Otorhinolaryngol 1999; 47:253-259

9. Hsueh C, Hsueh S, Gonzalez-Crussi F, Lee T, Su J. Nasal chondromesenchymal hamartoma in children: report of 2 cases with review of the literature. Arch Pathol Lab Med 2001; 125:400403.

10. Kim B, Park S, Min HS, Rhee JS, Wang KC. Nasal chondromesenchymal hamartoma of infancy clinically mimicking meningoencephalocele. Pediatr Neurosurg 2004; 40:136-140.

11. Norman ES, Berhman S, Trupiano JK. Nasal chondromesenchymal hamartoma: report of a case and review of the literature. Pediatr Dev Pathol 2004; 7:517-520.

12. Shet T, Borges A, Nair C, Desai S, Mistry R. Two unusual lesions in the nasal cavity of infants: a nasal chondromesenchymal hamartoma and an aneurysmal bone cyst like lesion: more closely related than we think? Int J Pediatr Otorhinolaryngol 2004; 68:359-364

13. Ozolek JA, Carrau R, Barnes EL, Hunt JL. Nasal chondromesenchymal hamartoma in older children and adults. Arch Pathol Lab Med 2005; 129:1444-1450.

14. Johnson C, Nagaraj U, Esguerra J, Wasdahl D, Wurzbach D. Nasal chondromesenchymal harmartoma: radiographic and histopathologic analysis of a rare pediatric tumor. Pediatr Radiol 2007; 37:101-104. 